\title{
Consumo de drogas lícitas en estudiantes de enfermería de una universidad privada en Bogotá, Colombia
}

\author{
Marta Cecília López-Maldonado ${ }^{1}$ \\ Margarita Antonia Villar Luis² \\ Edilaine Cristina da Silva Gherardi-Donato ${ }^{3}$
}

\begin{abstract}
Se realizó un estudio de corte transversal con el fin de establecer el consumo de sustancias psicoactivas licitas en una muestra de 237 estudiantes de la carrera de enfermería de una Universidad privada de la ciudad de Bogotá, Colombia y relacionarla con las variables independientes del estudio: edad, sexo, estado civil, semestre, número de hijos, trabajo, convivencia. Se construyo un cuestionario para obtener información básica sobre: edad, género, estado civil, número de hijos, trabajo y convivencia. Se utilizó el cuestionario de Tolerancia de Fagerström que utiliza una escala de puntuación leve, media y alta, para evaluar la gravedad de la dependencia a la nicotina y el AUDIT versión para autoaplicación con una escala de puntuación que representa un grado bajo, medio y alto de dependencia del alcohol. Se garantizó la confidencialidad mediante el autodiligenciamiento de encuesta anónima. Se realizó un análisis de frecuencia y porcentaje simples. Como relevante en esta población se encontró que el consumo de tabaco es de $24 \%$ y el de alcohol de $82 \%$. El nivel de dependencia encontrado para tabaco fue $5(8,5 \%)$ estudiantes con dependencia leve, 42 (72\%) con dependencia media y 12 (20\%) con alta dependencia. Al relacionar consumo de tabaco y consumo de alcohol se encontró que el $98 \%$ de los estudiantes que fumaban consumían alcohol. Se encontró que es más alto el porcentaje de estudiantes que nunca beben en el grupo de no fumadores (23\%) que en el de fumadores (1.72\%).
\end{abstract}

Descriptores: Consumo de Bebidas Alcohólicas; Consumo de Productos Derivados del Tabaco; Estudiantes de Enfermería; Enfermería.

\footnotetext{
${ }^{1}$ Profesora, Facultad de Enfermería, Pontificia Universidad Javeriana, Bogotá, Colômbia. E-mail: mclopez@javeriana.edu.co.

2 Enfermera, Doctora en Enfermería. Profesor Titular, Escola de Enfermagem de Ribeirão Preto, Universidade de São Paulo, Centro Colaborador de la OMS para el Desarrollo de la Investigación en Enfermería, SP, Brasil. E-mail: margarit@eerp.usp.br.

${ }^{3}$ Enfermera, Doctora en Enfermería. Profesor Doctor, Escola de Enfermagem de Ribeirão Preto, Universidade de São Paulo, Centro Colaborador de la OMS para el Desarrollo de la Investigación en Enfermería, SP, Brasil. E-mail: nane@eerp.usp.br.
}

Correspondencia:

Margarita Antonia Villar Luis

Universidade de São Paulo. Escola de Enfermagem de Ribeirão Preto

Departamento de Enfermagem Psiquiátrica e Ciências Humanas

Av. dos Bandeirantes, 3900

Bairro: Monte Alegre

CEP: 14040-902 Ribeirão Preto, SP, Brasil

E-mail: margarit@eerp.usp.br 


\section{Consumo de drogas lícitas entre estudantes de enfermagem de uma universidade privada em Bogotá, Colômbia}

Estudo de coorte transversal que objetivou estabelecer o consumo de substâncias psicoativas lícitas, em amostra de 237 estudantes de enfermagem, em uma universidade privada de Bogotá, Colômbia, e relacionar esse consumo às variáveis independentes do estudo: idade, sexo, estado civil, semestre, número de filhos, trabalho e convívio. Foi utilizado o questionário de tolerância de Fagerstöm, para avaliar a gravidade da dependência da nicotina, e o AUDIT, para avaliar dependência ao álcool. A confidência dos dados foi garantida através do autopreenchimento do formulário anônimo. Foi realizada análise por frequência e porcentagem simples. Como relevância, salienta-se a prevalência do consumo de tabaco em $24 \%$ e do álcool em $82 \%$ da amostra. O nível de dependência para o tabaco foi de 5 (8,5\%) estudantes com dependência leve, 42 (72\%) com dependência média e 12 (20\%) com alta dependência. Ao relacionar consumo de tabaco e consumo de álcool foi encontrado que $98 \%$ dos estudantes que fumam consumem álcool. É maior a porcentagem de estudantes que nunca bebem entre os não fumantes $(23 \%)$ do que no grupo de fumantes $(1,72 \%)$.

Descritores: Consumo de Bebidas Alcoólicas; Consumo de Produtos Derivados do Tabaco; Estudantes de Enfermagem; Enfermagem.

\section{Licit Drugs Consumption Among Nursing Students at a Private University in Bogotá, Colombia}

This cross-sectional study aimed to establish the consumption of licit psychoactive substances in a sample of 237 nursing students at a private university in the city of Bogota, Colombia; and relate it with independent study variables: age, sex, marital status, semester, number of children, work and living together. Fagerström's Tolerance questionnaire was used to evaluate the gravity of nicotine dependence and the AUDIT to evaluate alcohol dependence. Confidentiality was guaranteed through the self administered Questionnaire and anonymous survey. Univariate analysis was used. Relevant data in this population was tobacco consumption in about $24 \%$ and alcohol in $82 \%$. The level of tobacco dependence corresponded to $5(8.5 \%)$ students with slight dependence, 42 (72\%) with average dependence and 12 (20\%) with high dependence. When relating tobacco and alcohol consumption, $98 \%$ of the students who smoke consume alcohol. The percentage of students who never drink was higher in the group of non smokers (23\%) than among smokers $(1.72 \%)$.

Descriptors: Alcohol Drinking; Consumption of Tobaco-Derived Products; Students, Nursing; Nursing.

\section{Introducción}

El consumo de sustancias psicoactivas se considera un problema mundial que repercute tanto en el sector social, económico y político como en el de salud. En el 2000 , el consumo de alcohol, era responsable del $4 \%$ de la carga mundial de mortalidad, tan importante como los estragos hechos por el tabaquismo(1-2).
Dada la alta prevalencia del consumo de alcohol y tabaco en Colombia y su incidencia en jóvenes, algunos estudios muestran el impacto del consumo en el aumento de problemáticas sociales como la violencia intrafamiliar, la baja productividad, los altos índices de muertes violentas, además del deterioro psicológico y físico que conlleva el abuso del alcohol(3-4). 
Una revisión sistemática de estudios internacionales sobre prevalencia y factores de riesgo por tabaquismo en adolescentes en América del Sur mostró que el aumento de prevalencia del consumo de tabaco entre adolescentes está directamente asociado a la edad. Mediante análisis multivariado se encontró un OR de 9,9 para tabaquismo en el grupo de 14 a 16 años y de 28,7 en el grupo de 17 a 19 años en relación con los jóvenes de 10 a 13 años. La adolescencia ha sido identificada como factor de riesgo para la dependencia del uso de $\operatorname{tabaco}^{(5)}$.

De acuerdo con estos estudios son los jóvenes quienes contribuyen de forma más significativa a las cifras de prevalencia de consumo anual y mensual de alcohol. Siendo este grupo importante para el desarrollo futuro del país, se considera fundamental estudiar la prevalencia con el fin de comprender este fenómeno social y desarrollar estrategias que permitan a la universidad contribuir al mejoramiento de las condiciones de salud de esta población.

A su vez los trabajos realizados sobre sustancias psicotrópicas en estudiantes Universitarios se han orientado principalmente al estudio de drogas ilícitas( ${ }^{(6)}$, sobre el uso indebido de drogas en estudiantes de medicina(7) y sobre el consumo inmoderado de alcohol como factor de predicción de la persistencia del consumo de tabaco en jóvenes ${ }^{(8)}$.

Otros estudios se han orientado a determinar factores asociados al consumo diario de cigarrillo en adolescentes estudiantes de nivel básico secundario( ${ }^{(9)} \mathrm{y}$ a investigar el consumo inmoderado de alcohol como factor de predicción de la persistencia del consumo de tabaco en jóvenes ${ }^{(8)}$. En intervenciones se ha discutido programas para fortalecer factores protectores que limitan el consumo de tabaco y alcohol(10).

De acuerdo con un estudio brasileño la población de interés en este estudio, o sea los estudiantes de enfermería, es un grupo con enfoque en investigaciones que envuelven universitarios ${ }^{(11)}$.

Todos estos estudios justifican el interés en la temática sobre la prevalencia de consumo de alcohol y tabaco entre estos estudiantes, considerándose como drogas licitas aquellas permitidas socialmente que contribuyen a la disminución de las expectativas de vida de un porcentaje importante de la población mundial y aumentan las tasas de muerte por enfermedad prevenible.

\section{Objetivo}

El objetivo de este estudio fue evaluar el consumo de tabaco y alcohol en estudiantes de enfermería de una universidad privada de Bogotá, en Colombia, a fin de contribuir para realizar un programa de intervención en esa población.

\section{Metodología}

\section{Tipo e Local de Estudio}

Se realizó un estudio descriptivo-exploratorio porque describe y explora la situación específica de un grupo de estudiantes de enfermería (jóvenes) de una institución universitaria ubicada en la ciudad de Bogotá, sobre el fenómeno del consumo de drogas licitas y establece relaciones entre algunas variables de consumo de alcohol y tabaco y características socio-demográfica.

\section{Muestra}

Con procedimientos de inclusión y exclusión participaron 237 estudiantes en el intervalo de edad de 16 a 37 años. Para calcular la proporción de estudiantes se tuvo en cuenta los 505 estudiantes que conformaron el universo, con un nivel de confianza del $95 \%$ y un error máximo tolerable de $5 \%$, una prevalencia de consumo de alcohol de $51,9 \%$ obtenida de la encuesta de uso de sustancias psicoactivas en adolescentes. Para ello se usó la fórmula de cálculo de tamaño de muestra por proporciones.

Para seleccionar a los estudiantes, sujetos de esta investigación, de tal forma que todos tuvieran la misma oportunidad de participar en el estudio, se distribuyó el total de la muestra en forma proporcional en los ocho semestres y en cada uno de ellos se utilizó un muestreo probabilístico sistemático.

\section{Recolección de Datos}

Se realizó una prueba piloto en los estudiantes de la misma universidad para determinar el tiempo de respuesta y verificar la comprensión de las preguntas de los instrumentos. Se siguieron tanto en la prueba piloto como en la aplicación de los instrumentos en la muestra, las normas de administración en términos de la explicación sobre el contenido de las preguntas, el porqué se realizan y la necesidad de responderlas adecuadamente.

Fueron utilizados los siguientes instrumentos:

- Cuestionario de Identificación Socio-Demográfico para obtener información básica sobre edad, género, estado civil, número de hijos, trabajo, convivencia y semestre del curso.

- Cuestionario de Tolerancia de Fagerström (CTF), que 
tiene por finalidad detectar el consumo de tabaco $y$ estimar el grado de dependencia de la nicotina(12). EI CTF es un instrumento mundialmente utilizado; contiene 6 ítems con una puntuación máxima de 10 puntos, siendo que la dependencia de la nicotina está clasificada en cinco grados: muy baja (0-2 puntos), baja (3-4 puntos), media (5 puntos), elevada (6-7 puntos) e muy elevada (8-10 puntos)(13).

- Cuestionario AUDIT para identificación de problemas relacionados al uso de alcohol. El AUDIT consiste de 10 preguntas que evalúan el consumo de alcohol en los últimos 12 meses. Una puntuación de 0 a 7 indica consumo de bajo riesgo o abstemios, de 8 a 15 puntos indica consumo de riesgo, de 16 a 19 puntos indica uso nocivo o consumo de alto riesgo y la probable dependencia se establece con una puntuación de 20 puntos o más(14).

\section{Consideraciones Éticas}

El proyecto fue aprobado por el Comité de Investigación y Ética de la Facultad de Enfermería de Bogotá de la Pontificia Universidad Javeriana. Se solicitó el consentimiento verbal informado a los participantes del estudio, que, debidamente informados, manifestaron consentimiento en participar.

\section{Análisis de los datos}

Para el procedimiento y análisis de la información se empleó el software SPSS para Windows. El análisis fue ejecutado por frecuencia y porcentajes simples.

\section{Resultados}

La muestra estuvo constituida por 237 estudiantes de la carrera de enfermería de una universidad privada localizada en la ciudad de Bogotá. Predominó el género femenino $(91,6 \%)$, el estado civil soltero $(87,8 \%)$, los estudiantes sin hijos $(81,4 \%)$, la convivencia con los padres $(72,1 \%)$ y los estudiantes que no tuvieron ocupación laboral $(65,4 \%)$ (Tabla 1$)$.

Los resultados de la aplicación de este instrumento mostraron que el $24,0 \%$ de los estudiantes consumía tabaco. De acuerdo con los puntajes que se han establecido para esta escala, se tiene el $8,5 \%$ de los estudiantes tenía una dependencia leve, el 71,2\% una dependencia media y el $20,3 \%$ una alta dependencia del tabaco. Del total de 58 fumadores el $90,0 \%$ eran mujeres y el 9,0\% eran hombres. Dos de los estudiantes fumadores no registraron si eran del género masculino o femenino.
Tabla 1 - Descripción de las variables sociodemográficas de los estudiantes, Bogotá-Colombia, 2007

\begin{tabular}{|c|c|c|}
\hline Variables $(n=237)$ & Frecuencia & Porcentaje \\
\hline \multicolumn{3}{|l|}{ Género } \\
\hline Hombres & 18 & 7,6 \\
\hline Mujeres & 217 & 91,6 \\
\hline En blanco & 2 & 0,8 \\
\hline \multicolumn{3}{|l|}{ Edad } \\
\hline 16 a 20 & 113 & 47,7 \\
\hline 21 a 30 & 116 & 48,9 \\
\hline 31 a 40 & 8 & 3,4 \\
\hline \multicolumn{3}{|l|}{ Estado Civil } \\
\hline Soltero & 208 & 87,8 \\
\hline Casado & 14 & 5,9 \\
\hline Divorciado & 1 & 0,4 \\
\hline Viudo & 1 & 0,4 \\
\hline Unión Libre & 8 & 3,4 \\
\hline En Blanco & 5 & 2,1 \\
\hline \multicolumn{3}{|l|}{ Hijos } \\
\hline Sin hijos & 193 & 81,4 \\
\hline 1 hijo & 24 & 10,2 \\
\hline 2 hijos & 6 & 2,5 \\
\hline 3 hijos & 6 & 2,5 \\
\hline En blanco & 8 & 3,4 \\
\hline \multicolumn{3}{|l|}{ Trabajo } \\
\hline Sin trabajo & 155 & 65,4 \\
\hline No trabaja & 80 & 33,8 \\
\hline En blanco & 2 & 0,8 \\
\hline \multicolumn{3}{|l|}{ Convivencia } \\
\hline Padres & 171 & 72,1 \\
\hline Cónyuge & 20 & 8,4 \\
\hline Amigos & 3 & 1,2 \\
\hline Otros familiares & 22 & 9,4 \\
\hline Compañero & 3 & 1,2 \\
\hline Sólo & 10 & 4,3 \\
\hline En blanco & 8 & 3,4 \\
\hline Total & 237 & 100,00 \\
\hline
\end{tabular}

Los resultados permitieron reconocer un consumo de tabaco de $24,0 \%$. El total de estudiantes no fumadores fue de $179(76,0 \%)$, de los cuales $13(7,0 \%)$ son hombres, 165 mujeres (92\%) y e1 0,5\% no respondió a la pregunta sobre género.

Según los puntajes establecidos para el AUDIT, el $88,0 \%$ de los estudiantes presentó bajo consumo de alcohol; el $10 \%$ presentó un nivel medio de problemas con alcohol, y el $2 \%$ de los estudiantes presentó un nivel alto de dependencia del alcohol.

Al indagar si alguna vez habían bebido, se encontró que $43(18,0 \%)$ estudiantes dijeron nunca haber bebido y $194(82,0 \%)$ haber bebido. De estos 106 (54,6\%) dijeron beber una o menos veces al mes; 76 (39,2\%) dijeron beber de 2 a 4 veces al mes; $11(5,7 \%)$ bebían 
2 o 3 veces a la semana y $1(0,5 \%)$ afirmaron beber 4 o más veces a la semana.

Una interpretación más detallada de la puntuación total se obtuvo a partir de los ítems del cuestionario, lo que permitió reconocer que 23 estudiantes tuvieron una puntuación en la pregunta 2 igual o mayor que 1 ; 25 estudiantes tuvieron esta misma puntuación en la pregunta 3 lo cual indica consumo en un nivel de riesgo.

Respeto a las puntuaciones por encima de 0 en las preguntas 4,5 y 6, que indican la presencia o el inicio de una dependencia del alcohol, 24 estudiantes tuvieron esta puntuación en la pregunta 4; 29 estudiantes en la pregunta 5 y 20 en la pregunta 6 . Los puntos obtenidos en las preguntas 7 a 10, que indican que ya se están experimentando daños relacionados con el alcohol, se observó que 15 estudiantes puntuaron más que 0 en la pregunta 7 y 15 en la pregunta 8 .

De los 58 estudiantes que fumaban, 5 eran hombres $(8,6 \%), 52$ mujeres $(89,6 \%)$ y $1(1,8 \%)$ no respondió a la pregunta de género. De los estudiantes que fumaban, uno $(1,8 \%)$ nunca bebió; $21(36,0 \%)$ bebían una o menos veces al mes; $29(50,0 \%)$ bebían 2 a 4 veces al mes; 6 bebían 2 a 3 veces a la semana $(10,4 \%)$ y uno bebía 4 o más veces a la semana $(1,8 \%)$. El total de estudiantes fumadores que consumía alcohol fue de 57 o sea el $98,3 \%$ de los fumadores consumían alcohol.

Los estudiantes que dijeron no ser fumadores fueron 179 (76,0\%): 13 hombres y 165 mujeres. De estos, $42(24,0 \%)$ nunca bebían y 137 consumían alcohol: 10 hombres (7,3\%) y 127 mujeres (92,7\%). De los que bebían, $47,5 \%$ dijeron beber una o menos veces al mes; el 26,3\% dijo beber de 2 a 4 veces al mes y $2,8 \%$ bebían 2 a 3 veces a la semana. No se encontró en este grupo ningún estudiante que señalara beber 40 más veces a la semana.

El total de los estudiantes de la muestra que había consumido alcohol son 194 (81,8\%). De estos 6,3\% son hombres y $75,1 \%$ mujeres. De los estudiantes hombres que constituyeron la muestra sólo 3 , señalaron nunca haber consumido alcohol, mientras que el número de mujeres que dijeron no haber consumido nunca alcohol fue de 39. En el total de la muestra el porcentaje de los estudiantes hombres que consumían alcohol fue de $83,3 \%$ y el de mujeres de $82,0 \%$.

Al relacionar el uso de alcohol y el uso de tabaco se encontró que era más alto el porcentaje de estudiantes que dijeron nunca ingerir alcohol en el grupo de no fumadores $(23,0 \%)$ que en el grupo de fumadores $(1,8 \%)$. Mientras que en el grupo de fumadores el
$35,0 \%$ bebían una o menos veces al mes, en el grupo de no fumadores este porcentaje fue de $48,33 \%$.

Cincuenta de los estudiantes que fumaban eran solteros $(86,2 \%) ; 3$ eran casados $(5,17 \%) ; 2$ vivían en unión libre $(3,44 \%) ; 2$ no respondieron $(3,44 \%)$ y 1 era separado $(1,72 \%)$. En el grupo de no fumadores 158 eran solteros $(88,26 \%) 11$ casados $(6,14 \%), 6$ unión libre $(3,35 \%) 1$ viudo $(0,55 \%)$ y 2 en blanco $(1,11 \%)$.

De los 194 que consumían alcohol 175 eran solteros (90\%); 9 casados $(4,63 \%) ; 1$ era viudo $(0,51 \%)$, 4 vivían en unión libre $(2,06 \%)$ y 3 estudiantes no respondieron $(1,54 \%), 2$ separados $(1,03 \%)$

De los estudiantes que fumaban 48 no tenían hijos $(83 \%) ; 8(14 \%)$ tenían un hijo y $1(1,72 \%)$ tenía 2 hijos, 1 no respondió $(1,72 \%)$. En este grupo se encontraban 57 de los estudiantes que consumían alcohol.

En el grupo de estudiantes que no fumaban 145 (81\%) no tenían hijos, 16 (9\%) un hijo; 5 (3\%) dos hijos, $6(3,35 \%)$ tres hijos. Se encontraban incluidos en este grupo los 137 estudiantes que consumían alcohol.

De los 58 estudiantes que dijeron fumar se encontró que $45(58,0 \%)$ vivían con sus padres, 4 (7,0\%) con su cónyuge; $5(9,0 \%)$ con otros familiares, 1 vivía solo $(1,7 \%)$, y 2 no respondieron $(3,4 \%)$. El porcentaje de estudiantes que dijeron no fumar y que vivían con sus padres fue igualmente alto y correspondió al 70\% de este grupo (126 estudiantes). Le siguen en su orden los 16 estudiantes que vivían con otros familiares $(9,0 \%)$ y los 14 estudiantes que vivían con su cónyuge $(8,0 \%)$. El restante vivían solos $9(5,0 \%), 3$ con amigos y 8 no respondieron $(4,5 \%)$.

El porcentaje de estudiantes que consumían alcohol y que vivían con sus padres fue muy alto $(84,2 \%)$; el $7,0 \%$ vivían con su cónyuge, el $1,5 \%$ con sus amigos, el $8,8 \%$ con otros familiares, el $5,1 \%$ solos y el $0,5 \%$ con compañeros.

El $62,0 \%$ de los estudiantes que fumaban no trabajaba, sólo lo hacían el 37,0\%. Igualmente en el grupo de no fumadores se encontró que el $65,0 \%$ de los estudiantes no trabajaba mientras que el 30,0\% si lo hacía. En este grupo 6 estudiantes no respondieron la pregunta $(3,3 \%)$.

En el grupo de los estudiantes que nunca habían ingerido alcohol (42) se encontró que 16 (38,0\%) trabajaban. En el grupo de los que consumían, 63 $(32,4 \%)$ trabajadores, $128(66,0 \%)$ no trabajadores, 3 $(1,5 \%)$ en blanco.

El $50 \%$ de los estudiantes que fumaban estaban en el intervalo de 16 a 20 años, le seguían con un porcentaje del 32,7\% los del grupo de 21 a 25 años, con 
un $10,3 \%$ los del grupo de 26 a 30 años y en el grupo de 31 y más el 6,8\%.

Al relacionar alcohol y edad se encontró que el $40,0 \%$ de los estudiantes que nunca bebían estaban en el grupo de 16 a 20 años. En igual porcentaje estaban los que bebían 1 o menos veces al mes. En el grupo que bebía 2 a 4 veces al mes el mayor porcentaje 61,7\% estaba en el intervalo de 16 a 20 años y el $80,0 \%$ de los que bebían 2 a 3 veces a la semana tenían igualmente de 16 a 20 años.

El mayor porcentaje de estudiantes fumadores estaba en el $6^{\circ}$ semestre con un porcentaje del 45,2\%. Le seguían el $1^{\circ}$ y 70 semestres con el $17,2 \%$ cada uno. Los menores porcentajes se ubicaron en el $6^{\circ}$ y 70 semestres con el 3,4\%.

El mayor porcentaje de estudiantes que nunca bebían se ubicaron en el $5^{\circ}$ semestre con un 30,0\%, seguido del $21,0 \%$ en los estudiantes del $8^{\circ}$ semestre. En los estudiantes del $8^{\circ}$ semestre se encontró un porcentaje de no bebedores de tan sólo el 4,8\%.

\section{Discusión}

En la estructura nacional de población por sexo en 2005, el mayor porcentaje de mujeres se encontraban en el intervalo de 20 a 24 años (5\%) mientras que en el intervalo de 35 y más se encontraban el $4 \%$ de la población femenina del país(15). A su vez, estas condiciones de la muestra se relacionan de una parte con la edad promedio de ingreso a la educación superior que en Colombia es de 17 años y de otra con el acceso de auxiliares de enfermería a la educación superior a partir de la política formulada por la Asociación Colombiana de Facultades de Enfermería(16). Política a la que se incorporó la facultad de enfermería en la que se realizó el estudio.

Predominó en este grupo el sexo femenino con 217 estudiantes $(91,0 \%)$ lo cual muestra el predominio histórico de la mujer en la educación superior de enfermería y se relaciona con el total de la población de la carrera que para 2007 era de 526 estudiantes, de las cuales 488 eran mujeres $(92,8 \%)$ y el restante 38 hombres $(7,2 \%)$. La incursión de los hombres a los programas de enfermería del nivel superior es relativamente reciente e se inicia en la facultad en la década de los 80 con un aumento progresivo, no significativo.

Los datos encontrados en el estudio se relacionan con los del Censo General realizado en 2005, en el que se observa que el mayor porcentaje de la población colombiana es soltera $(44,9 \%)$, además de que se relaciona con la edad dado que el $84,81 \%$ de la muestra de estudio es menor de 25 años ${ }^{(15)}$. Estos datos a su vez se relacionan con los encontrados en la variable convivencia.

En el grupo de estudio, el 80,0\% (193) no tenían hijos. Este mayor porcentaje de estudiantes sin hijos se relaciona con el mayor número de estudiantes menores de 25 años y muy posiblemente con su condición de estudiantes, lo cual centra sus intereses en terminar una carrera antes de constituir una familia y/o asumir otro tipo de responsabilidades que pueden de alguna manera limitar el cumplimiento de la meta de ser profesional. El número de hijos encontrados en el grupo de estudiantes de la muestra se relaciona a su vez con la tasa global de fecundidad en la zona urbana colombiana, que era de 2,65 hijos por mujer ${ }^{(15)}$.

Con relación a los trabajadores, el porcentaje puede corresponder a los estudiantes que se vinculan a la carrera como trabajadores del sector salud que optan por la estrategia de vincular estudio-trabajo como posibilidad de ascenso personal y profesional y que como ya se indicó, corresponde a una política nacional de acceso a la educación superior de enfermería de auxiliares de enfermería.

Sobre el uso de tabaco, llama la atención el gran porcentaje de estudiantes que consumen tabaco $(24,0 \%)$ en comparación con otros estudios que traen porcentajes mucho inferiores, entre el 2 y $9 \%$ aproximadamente ${ }^{(8,17)}$.

El consumo de alcohol al menos una vez en la vida y para las otras frecuencias de uso se equipararon con lo encontrado en otro estudio entre universitarios ${ }^{(8)}$.

\section{Conclusiones}

La prevalencia de tabaco en el grupo de estudiantes que constituyeron la muestra fue del $24,0 \%$ y la prevalencia de consumo de alcohol de 82,0\%. La prevalencia encontrada en este grupo es mayor que la descrita en la literatura.

El 50,0\% de los estudiantes fumadores están en el intervalo de edad de 16 a 20 años. En este mismo intervalo está el mayor porcentaje de estudiantes que dicen consumir alcohol.

El mayor porcentaje de fumadores se ubica en el 20 semestre y el mayor número de estudiantes que consumen alcohol está en el $6^{\circ}$ semestre.

El $24,3 \%$ de los estudiantes que consumen alcohol presentan de acuerdo con el dominio y contenido de los ítems del cuestionario AUDIT un nivel de riesgo: 
el $37,0 \%$ el inicio de una dependencia al alcohol y el $15,0 \%$ experimentan daños relacionados con el alcohol.

Frente a los resultados, se recomiendan realizar estudios de intervenciones en esta población a fines de reducir los daños del uso y de la dependencia de alcohol y tabaco a que están sujetos estos estudiantes.

\section{Agradecimientos}

Agradecemos a la Comisión Interamericana para el Control del Abuso de Drogas/CICAD de la Secretaria de Seguridad Multidimensional/SSM de la Organización de los Estados Americanos/OEA, la Secretaria Nacional de Políticas sobre Drogas/SENAD do Gabinete de Seguridad Institucional/Brasil, la Escuela de Enfermería de Ribeirao Preto de la Universidad de Sao Paulo y Centro Colaborador de la Organización Mundial de la Salud para el Desarrollo de la Investigación en Enfermería, la población representada en los estudios de investigación, bien como a las autoridades de las universidades representadas por los participantes del Programa En-Line de Especialización en Investigación sobre el Fenómeno de las Drogas, periodos 2006, 2007, 2008 y 2009.

\section{Referencias}

1. Organización Mundial de la Salud (OMS), (2004). Problemas de salud pública causados por el alcohol. Consejo Ejecutivo. [acceso 18 enero 2005]. Disponible en: http://www.who.int/gb.oms.org

2. Fagerstrom K. The epidemiology of smoking: health consequences and benefits of cessation. Drugs. 2002;62 Suppl 2:1-9.

3. Moral MV, Sirvent C, Rodríguez FJ. Comparación de la eficacia preventiva de cuatro programas de intervención psicosocial sobre las actitudes hacia el consumo de sustancias psicoactivas. Trastornos Adictivos. 2004;6:248-61.

4. Miller MA, Alberts JK, Hecht ML, Trost MR, Krizrk RL. Adolescent relationships and drug use. New York: Lawrence Erlbaum Associates; Inc. Publishers 2000.

5. Malcon MC, Menezes AMB, Maia MFS, Chatkin M, Victora CG. Prevalência e fatores de risco para tabagismo em adolescentes na América do Sul: uma revisão sistemática da literatura. Rev Panam Salud Publica/Pan Am J Public Health. 2003;13:222-8.

6. Gálvez E. Uso indebido de drogas en estudiantes de 6 años de medicina. Rev. Habanera de Ciencias Medicas noviembre-diciembre 2005;4(5).
7. Gonzáles, R. Consumo de sustancias en estudiantes de 5 año de medicina: Evolución de tres lustros. Rev. Hospital Psiquiátrico Habana. 2005;2(2).

8. Sánchez-Zamorano LM, Hernández-Avila M, LazcanoPonce E. El consumo inmoderado de alcohol como factor predictor de la persistencia del consumo de tabaco en jóvenes. Salud Publica Mex 2006; 48(supl 1):S41-7.

9. Martínez J. Factores asociados al consumo diario de cigarrillos en adolescentes, estudiantes de Bucaramanga. Biomédica (Bta) dic 2004; 25(4):518-26.

10. Esparza S. Programa para fortalecer factores protectores que limitan el consumo de Tabaco y Alcohol en estudiantes de educación media. Rev. Latino-Am Enfermagem. marzo-abril 2004;12(2):324-32.

11. Mardegan PS, Souza RS, Buaiz V, Siqueira MM. Uso de substâncias psicoativas entre estudantes de enfermagem. J Bras Psiquiatr. 2007;56(4):260-6.

12. Fagerström KO, Schneider NG. Measuring nicotine dependence: a review of the Fagerström Tolerance Questionnaire. J Behav Med. 1989;12:159-82.

13. Halty LS, Hüttner MD, Oliveira No IC, Santos VA, Martins G. Análise da utilização do Questionário de Tolerância de Fagerström (QTF) como instrumento de medida da dependência nicotínica. J. Pneumol. jul-ago 2002;28(4):180-6.

14. Babor TF, Fuente JR, Saunders J, Grant M. AUDIT The alcohol use disorders identification test: guidelines for use in primary health care. Geneva: WHO 1989.

15. Departamento Administrativo Nacional de Estadística (DANE), CO. Censo General 2005. Colombia; 2005.

16. Asociación Colombiana de Facultades de Enfermería (ACOFAEN). Exámenes de Estado de Calidad de la Educación Superior en Enfermería - ECAES. Bogotá, D.C.; 2004.

17. Ribeiro SA, Jardim JRB, Laranjeira RR, Alves AKS, Kesselring $F$, Fleissig L, Almeida MZH, Matsuda $M$, Hamamoto RS. Prevalência de tabagismo na Universidade Federal de São

Paulo, 1996 - dados preliminares de um programa institucional. Rev Ass Med Brasil. 1999;45(1):39-44. 